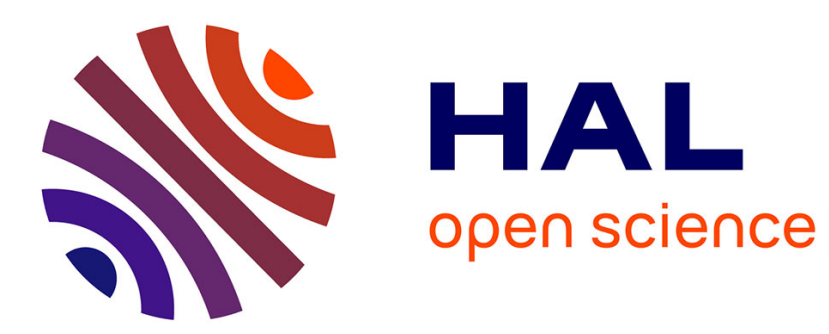

\title{
Control of Delayed Recycling Systems with an Unstable Pole at Forward Path
}

Juan Francisco Marquez Rubio, Basilio del Muro-Cuellar, Olivier Sename

\section{To cite this version:}

Juan Francisco Marquez Rubio, Basilio del Muro-Cuellar, Olivier Sename. Control of Delayed Recycling Systems with an Unstable Pole at Forward Path. ACC 2012 - American Control Conference, Jun 2012, Montréal, Canada. pp.5658-5663. hal-00937246

\section{HAL Id: hal-00937246 https://hal.science/hal-00937246}

Submitted on 28 Jan 2014

HAL is a multi-disciplinary open access archive for the deposit and dissemination of scientific research documents, whether they are published or not. The documents may come from teaching and research institutions in France or abroad, or from public or private research centers.
L'archive ouverte pluridisciplinaire HAL, est destinée au dépôt et à la diffusion de documents scientifiques de niveau recherche, publiés ou non, émanant des établissements d'enseignement et de recherche français ou étrangers, des laboratoires publics ou privés. 


\title{
Control of Delayed Recycling Systems with an Unstable Pole at Forward Path
}

\author{
J. F. Marquez Rubio, B. del Muro Cuéllar and Olivier Sename
}

\begin{abstract}
Unstable time delay system and recycling system pose a challenge problem in their own. When unstable time delay system have recycle the control problem becomes even more difficult. A control methodology for these systems is proposed in this paper. The strategy is based on the observation that if some internal system were available, then it would be possible to decouple the backward dynamics and then a feedback controller could be designed for the forward dynamics. The key for this strategy to be carried out is the asymptotic observerpredictor proposed to estimate the required internal signals. Necessary and sufficient conditions to assure convergence of this observer are given. Then, the strategy is complemented by using a PID controller with two degree of freedom in order to track step input references. Robustness to delay uncertainty is also discussed.
\end{abstract}

\section{INTRODUCTION}

In recycling system the output of a process is partially feedback to the input. Recycling processes reuse the energy and the partially processed matter increasing the efficiency of the overall process. They are commonly found in chemical industry, for instance, in a typical plant formed by reactor/separator process, where reactants are recycled back to the reactor [1].

Recycling processes are systems with positive feedback which can give rise to some undesirable effects. Particularly the so called snowball effect is observed in the operation of many chemical plants with recycle streams. Snowball means that a small change in a load variable causes a very large change in the flow rates around the recycle loop. Although snowballing is a steady state phenomenon and has nothing to do with dynamics, it depends on the control structure [2]. Disadvantages of snowball effect has drawn the attention of some researchers. Luyben [3], studied the effects of recycle loops on process dynamics and their implications to plantwide control. Scali and Ferrari [4], analyzed the problem under the concept of recycle compensation to recuperate inherent process dynamics, i.e. dynamics without recycle. Similar approaches were extended by Lakshminarayanan and Takada [5], and Kwok et. al [6]. It is known that when recycle loops and time delays occur, exponential terms appear in forward and backward paths. In state space representation recycled system with time delay correspond to systems with delays on the input and the state. Model approximation has been proposed to remove the exponential terms from the

J.F. Marquez and B. del Muro are with Instituto Politécnico Nacional, ESIME Unidad Culhuacan, Santa Ana 1000, Coyoacán, D.F., 04430, México, bdelmuro@yahoo.com, jfcomr23@yahoo.com.mx,

Olivier Sename is with GIPSA-lab, Control Systems Dpt ENSE3BP46, Domaine Universitaire, BP46 F-38402 Saint Martin dHres cedex, France, olivier.senamedgipsa-lab.grenoble-inp.fr transfer function denominator of a delayed system, such as the method of moments [7], and Pade approximations [8]. Other techniques, such as Taylor series expansion [9], and the seasonal time-series model [6], have been proposed to obtain an approximate model to represent recycle systems. In [10] it is proposed an approximate model to represent recycle systems by using discrete-time approach. In turn, such approximate models can be used for stability analysis or control design [11], [12], [4], [13]. A system with time delay and open-loop unstable poles is notably more difficult to control than a system with only open-loop stable poles. For instance, the classical Smith Predictor can not be used in this situation. Introducing recycle in such system would lead to a very difficult (although interesting) problem. Existing modified schemas to Smith Predictor can not directly be applied to this kind of systems. That is the reason why, to the authors best knowledge, recycle is not used in unstable plants with significant transport delay. To partially overcome this situation, in this work the problem of recycled system composed of a plant with an unstable pole and $m$ stable poles at direct loop and a stable system of order $n$ in the recycle loop is addressed. Note that the present work intends to deal with a more generalized recycling plant than the studied in [14] and [15], where unstable first order plant in forward loop is considered. Under these conditions, in this work it is proposed an observer-predictor based control schema in order to stabilize and control recycling systems with delay term in both paths. Neccesary and sufficient conditions with respect to time delay size at direct path are given for the existence proposed observer. Then, estimated internal variables are used to eliminate the dynamics of recycle loop and design a control strategy achieving step tracking.

In Section II the problem is formulated and the class of systems considered in this work is precised. The general idea of the solutions is also outlined in this Section. Section III presents the control proposed. In Section IV, a robustness analysis is provided. Some simulations results are described in Section V. Such results illustrate the performance of the control here proposed. Finally Section V presents some conclusions.

\section{PROBLEM FORMULATION}

Consider the class of recycling system shown in Fig. 1, which can be described as,

$$
Y(s)=\left[\begin{array}{ll}
G_{d} & G_{d} G_{r}
\end{array}\right]\left[\begin{array}{l}
U(s) \\
Y(s)
\end{array}\right],
$$


with,

$$
\begin{aligned}
G_{d} & =G_{1}(s) e^{-\tau_{1} s}=\frac{\alpha}{(s-a)(s+b)} e^{-\tau_{1} s}, \\
G_{r} & =G_{2}(s) e^{-\tau_{2} s}=\frac{N(s)}{D(s)} e^{-\tau_{2} s},
\end{aligned}
$$

where $G_{d}(s)$, and $G_{r}(s)$ are transfer functions of the forward (or direct) and backward (or recycle) paths, respectively; $\tau_{1}, \tau_{2} \geq 0$ are the time delays associated to $G_{d}(s)$, and $G_{r}(s) . a, b \in \mathbb{R}$, with $a, b>0$, that is $G_{d}$ is unstable; $N(s)$ and $D(s)$ are polynomials on the complex variable $s . U(s)$ is the process input and $Y(s)$ is the process output.

For simplicity of the presentation it is considered a second order system with time delay at forward loop (given as in (2a)) of the recycling plant. However, results with respect to the generalized system at forward path (i.e., $G_{d}$ being a plant with an unstable pole, $m$ stable poles and time delay) are also presented.

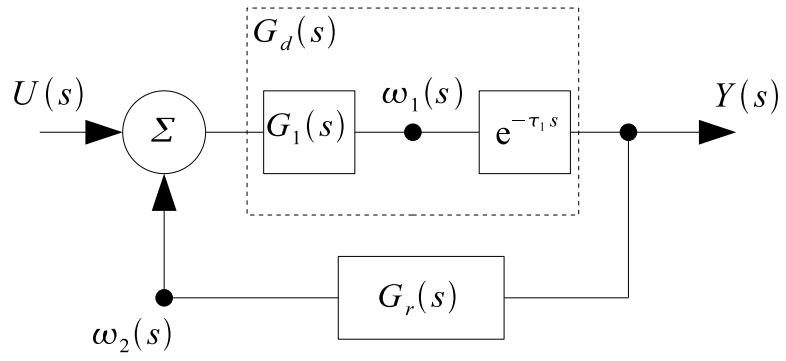

Fig. 1. A process with recycle by,

The closed-loop transfer function of system (1) is given

$$
G_{t}(s)=\frac{Y(s)}{U(s)}=\frac{D(s) \alpha e^{-\tau_{1} s}}{(s-a)(s+b) D(s)-\alpha N(s) e^{-\left(\tau_{1}+\tau_{2}\right) s}} .
$$

Note that exponential terms appear explicitly in numerator and denominator of $G_{t}(s)$. Stability of (3) is determined by the roots of its characteristic equation,

$$
Q(s)=(s-a)(s+b) D(s)-\alpha N(s) e^{-\left(\tau_{1}+\tau_{2}\right) s}=0 .
$$

More precisely, the overall path $U(s) \rightarrow Y(s)$ is stable if and only if all the roots of $Q(s)$ are contained in the open lefthalf complex plane. It is well known that the transcendental term in $Q(s)$ induces an infinite number of roots preventing the use of classical control design techniques and stability analysis methods.

Let us describe some ideas behind the methodology proposed. With reference to Fig. 1, if signal $\omega_{2}$ were known, then we could set, $U(s)=R_{1}(s)-\omega_{2}(s)$, obtaining the system shown in Fig. 2. Then it would be possible to design $R_{1}(s)$ as $R_{1}(s)=\left(R(s)-\omega_{1}(s)\right) J(s)$ like in Fig. 3. Since $\omega_{1}$ and $\omega_{2}$ are internal system signals an observerpredictor scheme to estimate these variables is developed in the following section.

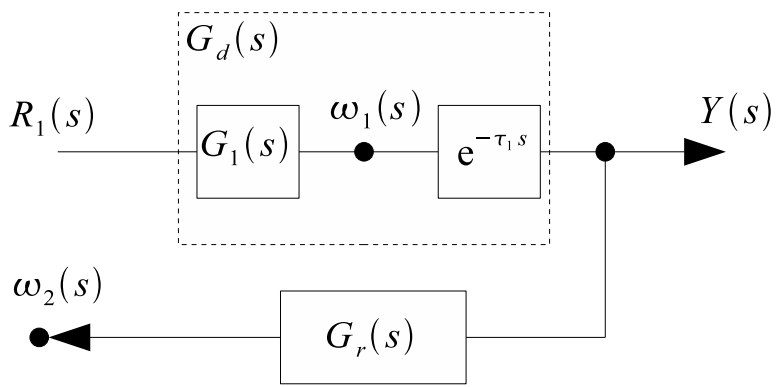

Fig. 2. System of Fig. 1 after applying the proposed U(s)

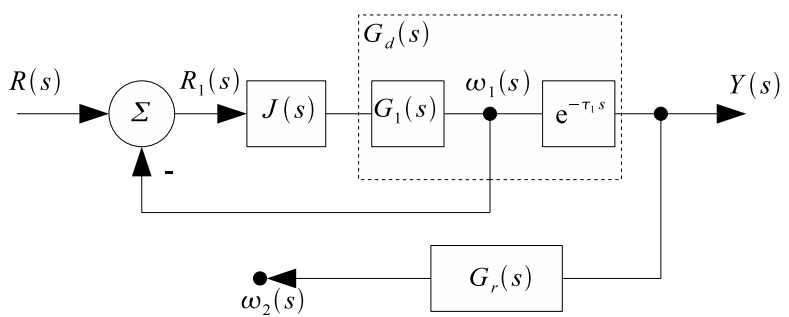

Fig. 3. A control structure for the system of Fig. 2

\section{OBSERVER-PREDICTOR BASED CONTROL}

\section{A. Preliminary stability results}

Consider the unstable input delay system,

$$
\frac{Y(s)}{U(s)}=G(s) e^{-\tau s}=\frac{\beta}{s-a} e^{-\tau s}
$$

with $a>0$. As a preliminary result, it is stated the existence conditions of a static output feedback,

$$
U(s)=R(s)-k Y(s),
$$

that ensures the stability of the closed loop system.

Lemma 1: [16] Let us consider the system (5) and the proportional control (6). There exist a gain $k$ such that the closed loop system,

$$
\frac{Y(s)}{R(s)}=\frac{\beta e^{-\tau s}}{s-a+k \beta e^{-\tau s}},
$$

is BIBO stable if and only if $\tau<\frac{1}{a}$.

The proof of this result can be easily obtained by considering different approaches as one based on the classical frequency domain. An alternative simple proof based on a discrete time approach is presented in [16]. The following results are similar to Lemma 1, but for a class of unstable delayed systems more complex than the previous one.

Lemma 2: [17] Consider the system,

$$
G(s)=\frac{\alpha}{(s-a)(s+b)} e^{-\tau s}
$$

with $a, b>0$ and $\frac{1}{a}-\frac{1}{b}>0$. Then, there exist a gain $k$ such that the closed loop system,

$$
\frac{Y(s)}{R(s)}=\frac{\alpha e^{-\tau s}}{(s-a)(s+b)+k \alpha e^{-\tau s}},
$$

is BIBO stable if and only if $\tau<\frac{1}{a}-\frac{1}{b}$. 
Note that the necessary condition $\frac{1}{a}-\frac{1}{b}>0$ in Lemma 2 is derived from a stability analysis of the transfer function (8) when $\tau=0$.

Proof: Let us consider the Lemma 1. There is a gain $k$ such that the system $\frac{\beta}{s-a} e^{-\tau s}$ is closed loop stable if and only if $\tau<\frac{1}{a}$. An analysis in the frequency domain confirms this result. Fig. 4 shows the Nyquist diagram for a system satisfying $\tau<\frac{1}{a}$. The Nyquist stability criterion states that when the loop is closed with a gain $k$, the system will be stable iff $0=N+P$, with $P$ the number of poles $G(s)$ in the right half plane and $N$ the number of clockwise round trips to the point -1 ( $N$ negative in counterclockwise) in the Nyquist diagram. In this case there is a gain that stabilizes the system since there is one tour counterclockwise to the point -1 . When $\tau<\frac{1}{a}$ is not satisfied, there is not detour in counterclokwise. The phase as a function of frequency $\omega$ is given by $\angle G(j \omega)=-\left(\pi-t g^{-1} \frac{\omega}{a}\right)-\omega \tau$. It can be shown that the condition $\tau<\frac{1}{a}$ is equivalent to ask that the angle path tap at least one point (for some frequency) with a value exceeding $-\pi$, that is $\angle G(j \omega)>-\pi$. Let us now analyze the system under consideration given by (7). It is evident that with $\tau<\frac{1}{a}$ and the parameter $b$ large enough there is a $k$ that stabilizes the system, since the Nyquist condition remains the same (one counterclockwise detour to the point $-1)$. Now we have,

$$
\angle G(j \omega)=-\left(\pi-t g^{-1} \frac{\omega}{a}\right)-t g^{-1} \frac{\omega}{b}-\omega \tau .
$$

Decreasing the value of parameter $b$ (always satisfying $\frac{1}{a}-$ $\frac{1}{b}>0$ ), the bound that forms the trajectory conterclockwise also decreses until extinction (See Fig. 5). As for small frequencies $t^{-1} \omega \varphi \approx \omega \varphi$, starting from $\angle G(j \omega)>-\pi$ it is not difficult to conclude the relation $\tau<\frac{1}{a}-\frac{1}{b}$.

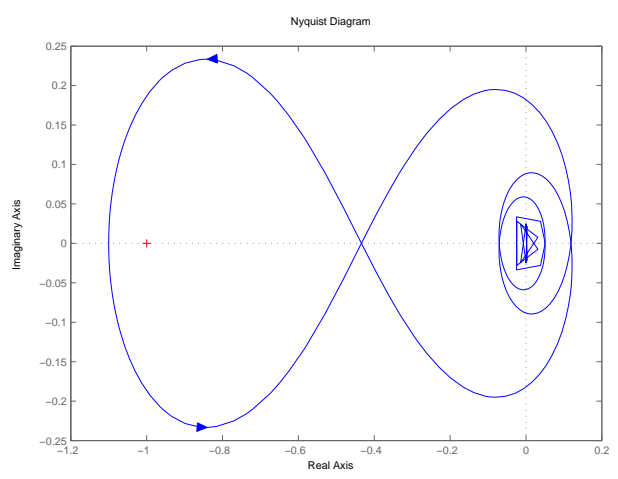

Fig. 4. Nyquist diagram when $\tau<\frac{1}{a}$

Lemma 3: [17] Consider the system,

$$
G(s)=\frac{\gamma}{(s-a)\left(s+b_{1}\right)\left(s+b_{2}\right) \ldots\left(s+b_{m}\right)} e^{-\tau s}
$$

with $m \in \mathbb{R}, a, b_{i}>0 \forall i=1,2, \ldots, m$; and $\frac{1}{a}-\sum_{i=1}^{m} \frac{1}{b_{i}}>0$. Then, there exist a gain $k$ such that the closed loop system,

$$
\frac{Y(s)}{R(s)}=\frac{\gamma e^{-\tau s}}{(s-a)\left(s+b_{1}\right)\left(s+b_{2}\right) \ldots\left(s+b_{m}\right)+k \gamma e^{-\tau s}},
$$

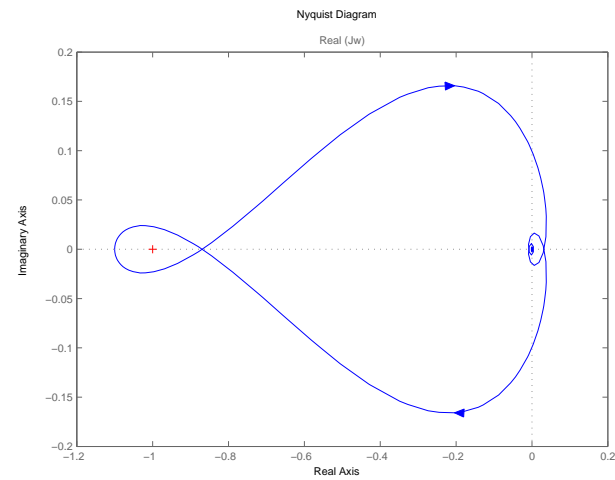

Fig. 5. Nyquist Diagram when rising the $b$ value.

is stable if and only if $\tau<\frac{1}{a}-\sum_{i=1}^{m} \frac{1}{b_{i}}$.

Proof: Consider the analysis presented in the proof of Lemma 2. Then if we consider now $m=2$, under the assumption $\tau<\frac{1}{a}-\frac{1}{b_{1}}-\frac{1}{b_{2}}$, and with $b_{2}$ large enough, there is $k$ that stabilizes the system (Nyquist condition remains one counterclockwise loop to the point -1 ). We have now,

$$
\angle G(j \omega)=-\left(\pi-t g^{-1} \frac{\omega}{a}\right)-t g^{-1} \frac{\omega}{b_{1}}-t g^{-1} \frac{\omega}{b_{2}}-\omega \tau .
$$

Now, decreasing the value of parameter $b_{2}$ (always satisfying $\left.\frac{1}{a}-\sum_{i=1}^{m} \frac{1}{b_{i}}>0\right)$, the loop that forms the detour counterclockwise decreses until extinction. Again considering $\operatorname{tg}^{-1} \omega \varphi \approx$ $\omega \varphi$, from $\angle G(j \omega)>-\pi$ it is not difficult to conclude the relation $\tau<\frac{1}{a}-\frac{1}{b_{1}}-\frac{1}{b_{2}}$. This analysis can be generalizated to any $m \in \mathbb{R}$ concluding that there is a gain $k$ such that the closed loop system given by (10) is BIBO stable if and only if $\tau<\frac{1}{a}-\sum_{i=1}^{m} \frac{1}{b_{i}}$

\section{B. Prediction Strategy}

In order to estimate $\omega_{1}$ and $\omega_{2}$ in Fig. 1 we propose the observer-predictor depicted in Fig. 6. Its convergence is established in the following result.

Theorem 4: Consider the observer-predictor scheme shown in Fig. 6, with $G_{r}$ a stable transfer function. There exists constant $k$ such that $\lim _{t \rightarrow \infty}\left[\omega_{i}-\widehat{\omega}_{i}\right]=0$ for $i=1,2$, if and only if $\tau_{1}<\frac{1}{a}-\frac{1}{b}$.

Proof: A state space representation of the observerpredictor scheme shown in Fig. 6 is

$$
\begin{aligned}
& \dot{\mathbf{x}}(t)=A \mathbf{x}(t)+A_{1} \mathbf{x}\left(t-\tau_{1}\right)+A_{2} \mathbf{x}\left(t-\tau_{2}\right)+B u(t) \\
& \mathbf{y}(t)=C \mathbf{x}\left(t-\tau_{1}\right)
\end{aligned}
$$

with, $\mathbf{x}(t)=\left[\begin{array}{llll}x_{d}(t) & x_{r}(t) & \widehat{x}_{d}(t) & \widehat{x}_{r}(t)\end{array}\right]^{T}, \mathbf{y}(t)=$ $\left[\begin{array}{ll}y(t) & \widehat{y}(t)\end{array}\right]^{T}, B=\left[\begin{array}{cccc}B_{d} & 0 & B_{d} & 0\end{array}\right]^{T}$, 


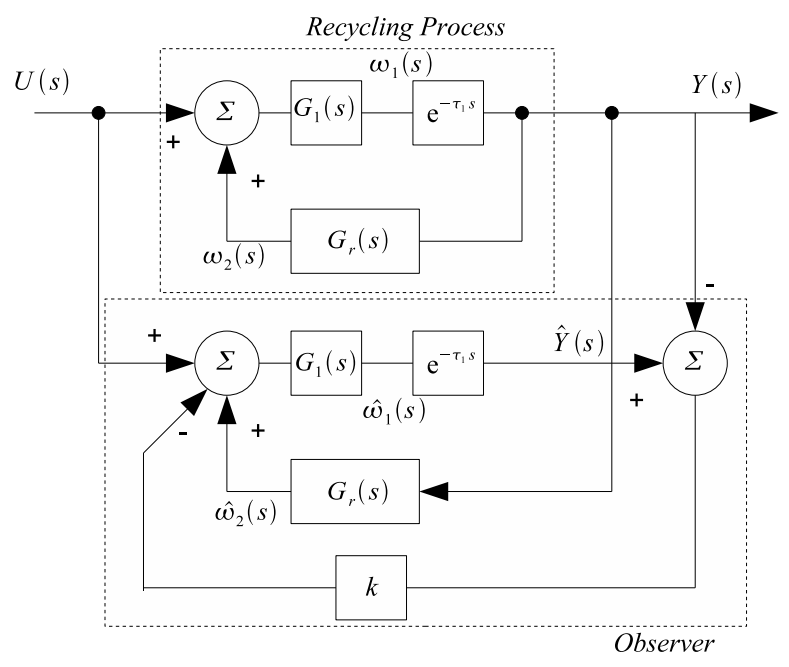

Fig. 6. Proposed observer schema

$\begin{aligned} A & =\left[\begin{array}{cccc}A_{d} & 0 & 0 & 0 \\ 0 & A_{r} & 0 & 0 \\ 0 & 0 & A_{d} & 0 \\ 0 & 0 & 0 & A_{r}\end{array}\right], \\ A_{1} & =\left[\begin{array}{cccc}0 & 0 & 0 & 0 \\ B_{r} C_{d} & 0 & 0 & 0 \\ B_{r} k C_{d} & 0 & -B_{d} k C_{d} & 0 \\ B_{r} C_{d} & 0 & 0 & 0\end{array}\right], \\ A_{2} & =\left[\begin{array}{cccc}0 & B_{d} C_{r} & 0 & 0 \\ 0 & 0 & 0 & 0 \\ 0 & 0 & 0 & B_{d} C_{r} \\ 0 & 0 & 0 & 0\end{array}\right], C=\left[\begin{array}{cccc}C_{d} & 0 & 0 & 0 \\ 0 & 0 & C_{d} & 0\end{array}\right],\end{aligned}$

where $\mathbf{x} \in \mathbb{R}^{p}$ (where $p=n+m+1$ ) is the state vector, $u \in \mathbb{R}$ is the input, $\mathbf{y} \in \mathbb{R}^{2}$ is the output, $\tau_{1} \geq 0$ and $\tau_{2} \geq 0$ are the time delays present in the system. $A_{d} \in \mathbb{R}^{(m+1) \times(m+1)}, B_{d} \in \mathbb{R}^{(m+1) \times 1}$, and $C_{d} \in \mathbb{R}^{1 \times(m+1)}$ are matrices and vectors parameters that corresponds to the forward loop in the process, and $A_{r} \in \mathbb{R}^{n \times n}, B_{r} \in \mathbb{R}^{n \times 1}$, and $C_{r} \in \mathbb{R}^{1 \times n}$ are matrices and vectors parameters that corresponds to recycling (or backward) path in the process, $\widehat{x}(t)$ is the estimation of $x(t)$. Note that for this analysis $m=1$ is considered.

Defining the state prediction errors $e_{x_{d}}(t)=\widehat{x}_{d}(t)-x_{d}(t)$, $e_{x_{r}}(t)=\widehat{x}_{r}(t)-x_{r}(t)$, and the output estimation $e_{y}(t)=$ $\widehat{y}(t)-y(t)$, it is possible to describe the behavior of the error signals as,

$$
\left[\begin{array}{c}
\dot{e}_{x_{d}}(t) \\
\dot{e}_{x_{r}}(t) \\
e_{y}\left(t+\tau_{1}\right) \\
e_{\omega_{2}}\left(t+\tau_{2}\right)
\end{array}\right]=A_{p}\left[\begin{array}{c}
e_{x_{d}}(t) \\
e_{x_{r}}(t) \\
e_{y}(t) \\
e_{\omega_{2}}(t)
\end{array}\right]
$$

with,

$$
A_{p}=\left[\begin{array}{cccc}
A_{d} & 0 & -B_{d} k & B_{d} \\
0 & A_{r} & 0 & 0 \\
C_{d} & 0 & 0 & 0 \\
0 & C_{r} & 0 & 0
\end{array}\right] .
$$

Note that $e_{y}(t)=C_{d} e_{x_{d}}\left(t-\tau_{1}\right)$ and that $e_{\omega_{2}}(t)=C_{r} e_{x_{r}}(t-$ $\left.\tau_{2}\right)$. Then, system (13) can be rewritten as,

$$
\begin{aligned}
\dot{e}_{x_{d}}(t)= & A_{d} e_{x_{d}}(t)-B_{d} k C_{d} e_{x_{d}}\left(t-\tau_{1}\right) \\
& +B_{d} C_{r} e_{x_{r}}\left(t-\tau_{2}\right), \\
\dot{e}_{x_{r}}(t)= & A_{r} e_{x_{r}}(t) .
\end{aligned}
$$

Since $A_{r}$ is a Hurwitz matrix, the stability of system (14) can be analyzed by considering the partial dynamics

$$
\dot{e}_{x_{d}}(t)=A_{d} e_{x_{d}}(t)-B_{d} k C_{d} e_{x_{d}}\left(t-\tau_{1}\right),
$$

or equivalently,

$$
\left[\begin{array}{c}
\dot{e}_{x_{d}}(t) \\
e_{y}\left(t+\tau_{1}\right)
\end{array}\right]=\left[\begin{array}{cc}
A_{d} & -B_{d} k \\
C_{d} & 0
\end{array}\right]\left[\begin{array}{c}
e_{x_{d}}(t) \\
e_{y}(t)
\end{array}\right] .
$$

Consider now a state space realization of system (9). It is easy to see that this dynamics can be written in state space form as,

$$
\left[\begin{array}{c}
\dot{x}(t) \\
y\left(t+\tau_{1}\right)
\end{array}\right]=A_{f}\left[\begin{array}{c}
x_{d}(t) \\
y(t)
\end{array}\right]+\left[\begin{array}{c}
B_{d} \\
0
\end{array}\right] u(t)
$$

with,

$$
A_{f}=\left[\begin{array}{cc}
A_{d} & -B_{d} k \\
C_{d} & 0
\end{array}\right] .
$$

Comparing (17) and (16) it is clear that Lemma 2 can be applied to system (16). Hence the result of the theorem follows.

Note that the proposed prediction strategy can be generalizated to recycling systems with an unstable pole and $m$ stable poles at forward loop,

$$
G_{d}=\frac{\alpha}{(s-a)\left(s+b_{1}\right)\left(s+b_{2}\right) \ldots\left(s+b_{m}\right)} e^{-\tau_{1} s} .
$$

In such case, the convergence of the observer is assured with the following result.

Theorem 5: Consider the observer-predictor scheme shown in Fig. 6, with $G_{r}$ a stable transfer function and $G_{d}$ defined as in (18). There exists constant $k$ such that

$$
\lim _{t \rightarrow \infty}\left[\omega_{i}-\widehat{\omega}_{i}\right]=0, \text { for } i=1,2,
$$

if and only if $\tau_{1}<\frac{1}{a}-\sum_{i=1}^{m} \frac{1}{b_{i}}$.

Proof: The proof of this Lemma can be stated in a similar way that the proof of Lemma 4, by using Lemma 3 instead of Lemma 2

\section{Proposed Control Scheme}

Having assured that estimated internal signals $\widehat{\omega}_{1}$ and $\widehat{\omega}_{2}$ converge to the original, the ideas depicted in Section II can be implemented. In this way, the control law can be depicted as, $U(s)=J(s)\left(R(s)-\widehat{\omega}_{1}(s)\right)-\widehat{\omega}_{2}(s)$. The complete control scheme is proposed in Fig. 7. 


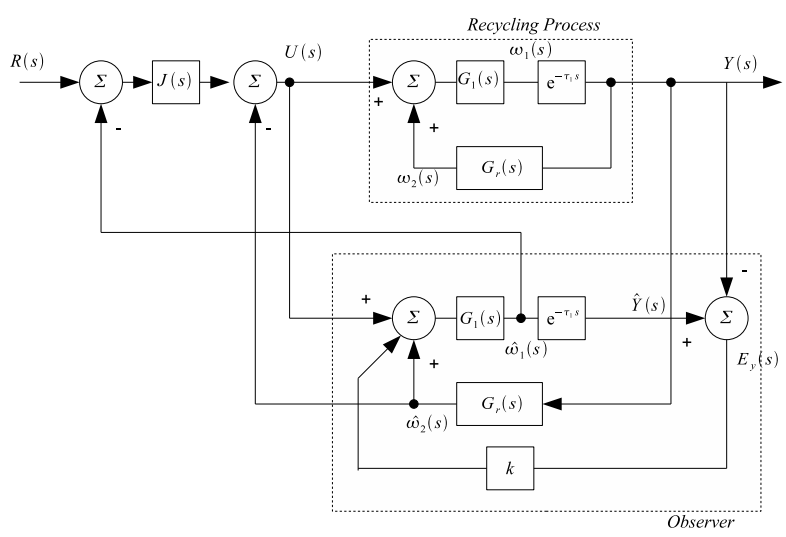

Fig. 7. Proposed control schema

\section{ROBUSTNESS WITH RESPECT TO TIMES DELAY UNCERTAINTIES}

A control strategy has been presented and knowledge of the real process is considered. However, in practice, the control strategy should take into account a robustness analysis. In this case, robustness analysis is done using procedure prosposed in [18]. This analysis is applied under the assumption that the observer and controller for the nominal case have been designed. Consider a state representation of the open-loop system with recycle in the nominal case (which can be obtained from the complete state representation of system-observer expressed in equation (11)),

$$
\begin{aligned}
\dot{x} & =\bar{A} x+\overline{A_{1}} x\left(t-\tau_{1}\right)+\overline{A_{2}} x\left(t-\tau_{2}\right)+\bar{B} u(t) \\
y & =\overline{C_{1}} x\left(t-\tau_{1}\right)
\end{aligned}
$$

Where $x=\left[\begin{array}{ll}x_{d} & x_{r}\end{array}\right]^{T} \bar{A}=\left[\begin{array}{cc}A_{d} & 0 \\ 0 & A_{r}\end{array}\right], \overline{A_{1}}=$ $\left[\begin{array}{cc}0 & 0 \\ B_{r} C_{d} & 0\end{array}\right], \overline{A_{2}}=\left[\begin{array}{cc}0 & B_{d} C_{r} \\ 0 & 0\end{array}\right], \bar{B}=\left[\begin{array}{c}B_{d} \\ 0\end{array}\right], \overline{C_{1}}=$ $\left.C_{d} \quad 0\right]$.

Before define perturbations for the recycling system, as an example, let the nominal system be,

$$
\dot{x}=A x(t)+B x(t-\tau),
$$

and the actual system as above but with $\tau$ replaced by $\tau_{0}$, then $p(x(t))=B\left[x(t-\tau)-x\left(t-\tau_{0}\right)\right]$. In this way, with $\theta=\tau_{0}-\tau$, we get,

$$
P_{1}(s, \theta)=B\left[e^{-\tau_{0} s}-e^{-\tau s}\right]=B e^{-\tau s}\left[e^{-\theta s}-1\right] .
$$

Based on previous developments, let define the perturbations on both times delay and matrix $\left(\bar{A}, \bar{A}_{1}\right.$ and $\left.\bar{A}_{2}\right)$ as,

$$
\begin{aligned}
& P(s, \theta, \delta)= \bar{A}_{\delta}+e^{-s \tau_{1}}\left[\overline{A_{1}}\left(e^{-s \theta_{1}}-1\right)+\overline{A_{1}} \delta_{1} e^{-s \theta_{1}}\right]+ \\
& e^{-s \tau_{2}}\left[\overline{A_{2}}\left(e^{-s \theta_{2}}-1\right)+\overline{A_{2}} \delta_{2} e^{-s \theta_{2}}\right], \\
& Q(s, \theta)=\overline{C_{1}} e^{-s \tau_{1}}\left(e^{-s \theta_{1}}-1\right) .
\end{aligned}
$$

where, $\bar{A}, \overline{A_{1}}$ and $\overline{A_{2}}$ are the nominal matrix of the recycle system and the corresponding uncertainties are given by $\bar{A}_{\delta}$,
$\overline{A_{1}} \delta_{1}$ and $\overline{A_{2}} \delta_{2}$. Then, a state space representation of the controller-observer shown in Fig. 7 can be obtained as,

$$
\begin{aligned}
\dot{\mathrm{x}}(\mathrm{t})= & \mathcal{A x}(\mathrm{t})+\mathcal{A}_{1} \mathrm{x}\left(\mathrm{t}-\tau_{1}\right)+\mathcal{A}_{2} \mathrm{x}\left(\mathrm{t}-\tau_{2}\right) \\
& +\mathcal{A}_{3} \mathrm{x}\left(\mathrm{t}-\theta_{1}\right)+\mathcal{A}_{4} \mathrm{x}\left(\mathrm{t}-\theta_{2}\right)
\end{aligned}
$$

with $\mathrm{x}(\mathrm{t})=\left[\begin{array}{ll}e_{x}(t) & x(t)\end{array}\right], e_{x}(t)=\widehat{x}(t)-x(x)$,

$$
\begin{aligned}
\mathcal{A} & =\left[\begin{array}{cc}
\bar{A} & 0 \\
\bar{B} K & \bar{A}-\bar{B} K
\end{array}\right], \mathcal{A}_{1}=\left[\begin{array}{cc}
\bar{A}_{1}-G \bar{C}_{1} & 0 \\
0 & \bar{A}_{1}
\end{array}\right], \\
\mathcal{A}_{2} & =\left[\begin{array}{cc}
\bar{A}_{2} & 0 \\
\bar{B} L & \bar{A}_{2}-\bar{B} L
\end{array}\right], \mathcal{A}_{3}=\left[\begin{array}{cc}
0 & \bar{A}_{1}-G \bar{C}_{1} \\
0 & \bar{A}_{1}
\end{array}\right], \\
\mathcal{A}_{4} & =\left[\begin{array}{ll}
0 & \bar{A}_{2} \\
0 & \bar{A}_{2}
\end{array}\right], G=\left[\begin{array}{lll}
k & 0 & 1
\end{array}\right]^{T},
\end{aligned}
$$

$K=\left[\begin{array}{lll}0 & J & 0\end{array}\right], L=\left[\begin{array}{lll}0 & 0 & C_{r}\end{array}\right]$. The characteristic equation of the system (23), is given by,

$$
\begin{aligned}
\gamma= & \operatorname{det}\left[\begin{array}{cc}
s I-F+G \bar{C}_{1} e^{-\tau_{1} s} & G Q-P \\
-M & s I-F+M-P
\end{array}\right] \\
= & \operatorname{det}\left(s I-F+G \bar{C}_{1} e^{-\tau_{1} s}\right) \operatorname{det}(s I-F+M) \\
& \operatorname{det}\left(I+\psi^{-1} \Theta(s, \theta)\right)=0
\end{aligned}
$$

where $\gamma=\operatorname{det}\left(s I-\mathcal{A}-\mathcal{A}_{1} e^{-\tau_{1} s}-\mathcal{A}_{2} e^{-\tau_{2} s}\right), \quad F=$ $\bar{A}+\bar{A}_{1} e^{-\tau_{1} s}+\bar{A}_{2} e^{-\tau_{2} s}, M=\bar{B} K+\bar{B} L e^{-\tau_{2} s}$ and $\psi=$ $\left[\begin{array}{cc}s I-F+G \bar{C}_{1} e^{-\tau_{1} s} & 0 \\ -M & s I-F+M\end{array}\right]$ is the matrix corresponding to the combined observer-controller for nominal system, and $\Theta(s ; \theta)$ collects the plant uncertainty.

If the closed loop quasi polynomials $\operatorname{det}(s I-F+$ $\left.G \bar{C}_{1} e^{-\tau_{1} s}\right)$ and $\operatorname{det}(s I-F+M)$ are stable for a proper choice of $G, K$ and $L$ then the perturbed loop system remains stable if $\operatorname{det}\left(I+\psi^{-1} \Theta(s ; \theta)\right)$ does not change sign when $s$ sweeps the imaginary axis. This yields to the criterion,

$$
\begin{aligned}
p_{p} & =\operatorname{det}\left[I+\left[\begin{array}{ll}
\widetilde{Q}_{p q} & \widetilde{Q}_{p}
\end{array}\right]\left[\begin{array}{c}
G Q-P \\
-P
\end{array}\right]\right] \\
& =\operatorname{det}\left[I+N_{c}(s) D_{c}(s)\right],
\end{aligned}
$$

where,

$$
\begin{aligned}
\widetilde{Q}_{p q} & =(s I-F+M)^{-1} M\left(s I-F+G \bar{C}_{1} e^{-\tau_{1} s}\right)^{-1}, \\
\widetilde{Q}_{p} & =(s I-F+M)^{-1},
\end{aligned}
$$

which only depends on the nominal system and observer/controller parameter. By using Rouche's theorem, it follows that the condition for stability is,

$$
\left\|N_{c}(s) D_{c}(s, \theta)\right\|_{\infty}<1 .
$$

\section{Simulation Results}

In this section, an academic example show the performance of observer based control strategy previously proposed.

Example. Consider the recycled time delay system of the form (1) with,

$$
G_{d}=\frac{1}{(s-1)(s+10)} e^{-0.5 s}, \quad G_{r}=\frac{1}{s+1} e^{-2 s} .
$$

Following the procedure above described, it is obtained a proportional gain $k=12$. The free delay direct path can be 
stabilized by a two degree of freedom PID [19], obtaining a general feedback of the form, $U(s)=R(s) G_{f f}(s)-$ $G_{c}(s) \widehat{\omega}_{1}(s)-\widehat{\omega}_{2}(s)$, with $G_{f f}(s)=160\left(0.3+\frac{1.6}{s}+0.1 s\right)$ and $G_{c}(s)=160\left(1+\frac{1.6}{s}+0.1 s\right)$.

Fig. 8 shows the stability condition given by (24), where the uncertainties in time delays $\theta_{1}=0.005$ and $\theta_{2}=0.23$ are considered. In this case, such combination of uncertainties gives as result a stable closed loop system since $\left\|N_{c}(s) D_{c}(s, \theta)\right\|_{\infty}=0.9903<1$.

Now, in order to evaluate the output signal evolution some numerical simulations are presented. It is considered a positive unit step input and initial conditions in the process and the observer of magnitude 0.4 units and 0.2 units, respectively. In Fig. 9, a continuous line shows the output response when it is considered the exact knowledge of the model parameters; a dashed line presents the output signal when the time delays $\tau_{1}$ and $\tau_{2}$, are increased by $8 \%$ and $15 \%$, respectively. From Fig. 9 it can be seen the observer predictor convergence and the well behavior of the control based on estimated signals.

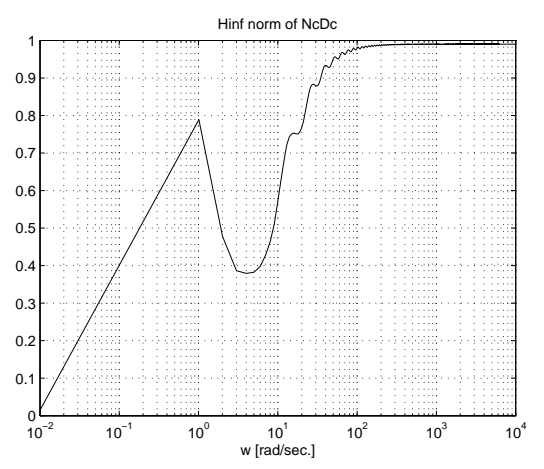

Fig. 8. $\left\|N_{c}(s) D_{c}(s, \theta)\right\|_{\infty}$ for $\theta_{1}=0.005$ and $\theta_{2}=0.23$

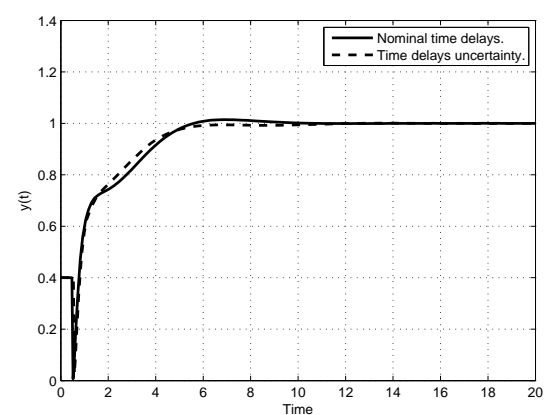

Fig. 9. Output signal with different initial condition in process and observer.

\section{CONCLUSIONS}

Using recycle in unstable processes with significant time delay leads to a challenging control problem. In this work this problem has been addressed for the general case of an unstable pole, $m$ stable poles with significant delay in the forward path. Explicit conditions for the construction of an stabilizing observer based controller scheme for such class of systems are presented. The observer-prediction strategy is used to estimate some internal variables of the process that are used to: i) remove the dynamics of backward loop in the recycling process and ii) design a stabilizing control law for the free delay model of the forward path. The basis provided in this work could be useful for extending the kind of system for which the recycle can be used. Particularly, the case of one unstable pole with several stable poles and zeros plus significant time delay in the forward path could be addressed.

\section{REFERENCES}

[1] W. L. Luyben, B. D. Tyreus and M. L. Luyben, Plantwide control, McGraw Hill, New York; 1999.

[2] M. L. Luyben and W. L. Luyben, Essentials of process control, McGraw-Hill, 1997.

[3] W. L. Luyben, Temperature control of autorefrigetater reactor, J. Process Control, vol. 9, 1999, pp 301.

[4] C. Scali and F. Ferrari, Performance of control systems based on recycle compensators in integrated plants, J. Process Control, vol. 9, 1999, pp 425.

[5] S. Lakshminarayanan and H. Takada, Empirical modelling of processes with recycle: some insights via case studies, Chem. Eng. Sci., vol. 56, 2001, pp 3327.

[6] K. E. Kwok, M. Chong-Ping and G. A. Dumont, Seasonal model based control of processes with recycle dynamics, Ind. Eng. Chem. Res., vol. 40, 2001, pp 1633.

[7] A. Papadourakis, M. F. Doherty and J. M. Douglas, Approximate dynamic models for chemical process systems, Ind. Eng. Chem. Res., vol. 28, 1989, pp 546-552.

[8] M. Malek-Zavarei and M. Jamshidi, Time-Delay systems. Analysis, optimization and applications, NorthHolland, The Netherlands, 1987.

[9] A. J. Hugo, P. A. Taylor and J. D. Wright, Approximate dynamic models for recycle systems, Ind. Eng. Chem. Res., vol. 35, 1996, pp 485-487.

[10] B. Del Muro-Cuellar, M. Velasco-Villa, H. Puebla and J. AlvarezRamirez, Model approximation for Dead-Time Recycling Systems, Ind. Eng. Chem. Res., vol. 44, 2005, pp 4336-4343.

[11] Y. K. Samyudia, K. Kadiman, O. L. Lee and I. T. Cameron, Gap metric based control of processes with recycle systems, Proccedings of ADChEM, Pisa-Italy, 2000, pp 497-502.

[12] K. J. Astrom, C. C. Hang and B. C. Lim, A new Smith predictor for controlling a process with an integrator and long dead time, IEEE Trans. Aut. Control, vol. 39(2), 1994, pp 343-345.

[13] L. Maza-Casas, M. Velasco-Villa and Ja. Alvarez-Gallegos, "On the state prediction of linear systems with time-delays in the input and the state", Proc. 38th. IEEE Conf. On Decision and Control, USA, December, 1999 , pp. 239-244.

[14] J.F. Marquez-Rubio, B. del Muro-Cuéllar, M. Velasco-Villa and D. Cortés-Rodríguez, "Observer scheme for linear recycling systems with time delays", American Control Conference, San Francisco, USA, 2011.

[15] J.F. Marquez-Rubio, B. del Muro-Cuéllar, M. Velasco-Villa, D. CortsRodrguez and O. Senamec, "Control of delayed recycling systems with unstable first order forward loop", J. Process Control (2012), doi:10.1016/j.jprocont.2012.02.002

[16] B. Del Muro-Cuellar, J.F. Marquez-Rubio, M. Velasco-Villa, J. Alvarez-Ramirez and H. Puebla, Stabilization and control of unstable first order linear delay systems, Internat. Conf. on Modelling, Shangai, 2008.

[17] Yebrail Pedraza Beltrán, Omar González Najera and B. del Muro Cuéllar, Stabilization of High Order Systems with Delay Using a Predictor Schema, 52nd. MWSCAS IEEE, Cancun Mex., 2009.

[18] E. Verriest, O. Sename and P. Pepe, "Robust observer controller for delay-differential system", Proc. of the IEEE International Conference on Desicion and Control, Las Vegas, USA, December, 2002.

[19] K. J. Astrom and T. Hagglund, PID Controllers, theory, design and tuning, International Society for Measurement and Control, New York, 1995. 